\title{
Promoter Hypomethylation and Expression of PLS3 in Human Sezary Lymphoma Cells
}

\author{
Elodie Begue $^{1 *}$, Laurence Michel ${ }^{1 *}$, Francette Jean-Louis ${ }^{1}$, Martine Bagot ${ }^{1,2}$ and \\ Armand Bensussan ${ }^{1}$ \\ ${ }^{1}$ UMR976, Skin Research Center and Universite Paris Diderot, Sorbonne Paris Cite, Hopital Saint-Louis, Paris, France \\ ${ }^{2}$ Assistance Publique-Hopitaux de Paris, Hopital Saint Louis, Paris, France
}

Received: November 28, 2013; Accepted: December 23, 2013; Published: December 26, 2013

*Corresponding authors: Laurence Michel, Skin Research Center and Universite Paris Diderot, Sorbonne Paris Cite, Hopital Saint-Louis, Paris, F-75475 Paris, France, E-mail: laurence.michel@inserm.fr

Elodie Begue, Skin Research Center and Universite Paris Diderot, Sorbonne Paris Cite, Hopital Saint-Louis, Paris, F-75475 Paris, France, E-mail: begueelodie@gmail.com

\begin{abstract}
Previous studies about the mechanism responsible for the regulation of PLS3 gene expression in cutaneous T cell lymphoma suggested a possible role of DNA methylation in the control of tissuespecific expression of PLS3. Using methylation-specific polymerase chain reaction adapted for quantitative real-time PCR, the present study investigated the methylation status in two human lymphocyte cell lines derived from tumor lymphocytes of patients with Sezary syndrome, HuT-78 cells which express PLS3 mRNA and SeAx cells that do not, and used well-established inhibitor of DNA methylation, the nucleoside analog 5-aza-2-deoxycytidine (5-aza-dC), to further determine its effect on PLS3 gene expression in both cell lines. Our results clearly revealed a quantitative relationship between the methylation status of the PLS3 CpG region and PLS3 expression in HuT-78 and SeAx Sezary cell lines.
\end{abstract}

Keywords: T-plastin; PLS3; Methylation; Sezary syndrome (SS); CTCL

\section{Introduction}

A molecular feature of Sezary syndrome (SS), the leukemic variant of cutaneous T-Cell Lymphomas (CTCL), is the abnormal gene expression of PLS3 in 3 out of 4 patients [1-9]. Currently, PLS3 is usually not expressed in normal T lymphocytes, although a recent study observed PLS3 transcription in PBLs from less than 5\% healthy individuals [10] in contrast to our own data [9]. Plastins, also known as fimbrins, are a family of actin-bundling proteins that are evolutionarily conserved from yeast to humans. In humans, three distinct isoforms have been identified and are expressed in a tissue-specific manner: I-plastin (PLS1) in intestinal and renal brush borders, L-plastin (LCP1) in hematopoïetic cells, and T-plastin (PLS3) in all other non hematopoietic tissues [11].

The mechanism responsible for the regulation of PLS3 gene expression remains to be investigated. Previous studies suggested a possible role of DNA methylation in the control of tissuespecific expression of PLS3 [12]. Actually, an inverse correlation between transcriptional activation and hypermethylation of local CpG sites has been reported for a number of genes in association with cell-type-specific expression and repression during tumorigenesis [13]. In CTCL, a recent work found no evidence for PLS3 mutations within coding or promoter regions, but showed significant hypomethylation of CpG dinucleotides 95-99 within the PLS3 CpG island restricted to the $\mathrm{PLS3}^{+}$population [8]. Using Methylation-Specific Polymerase (MSP) chain reaction adapted for quantitative real-time PCR (qRT-PCR), we investigated herein the methylation status in CTCL. We studied two human lymphocyte cell lines derived from tumor lymphocytes of patients with Sezary syndrome, HuT-78 cells which express PLS3 mRNA and SeAx cells that do not, for methylation status and used a wellestablished inhibitor of DNA methylation, the nucleoside analog 5-aza-2-deoxycytidine (5-aza-dC), to further determine its effect on PLS3 gene expression in both cell lines.

\section{Material and Methods}

\section{Cell cultures}

The Sezary cell line HuT-78 was purchased from European Collection of Animal Cell Cultures (ECACC, Salisbury, England). SeAx cell line established from a SS patient was kindly provided by Dr Keld Kaltoft (University of Aarhus, Denmark). The CTCL cell lines were grown at $37^{\circ} \mathrm{C}$ with $5 \% \mathrm{CO}_{2}$ at the concentration $5 \times 10^{4}$ cells $/ \mathrm{ml}$ in RPMI 1640 Glutamax $^{\mathrm{TM}}$-I medium, supplemented with $10 \%$ fetal bovine serum (FBS) and $100 \mathrm{U} / \mathrm{mL}$ penicillin, $100 \mu \mathrm{g} / \mathrm{mL}$ streptomycin, (GIBCO).

\section{Multiplex and quantitative Real-time PCR (qRT-PCR)}

RNA was isolated from cell lines using RNeasy Mini Kit according to the manufacturer's instructions with one step DNAse I (QIAGEN). Genomic DNA-free RNA was then converted into cDNA using the ThermoScript qRT-PCR system (INVITROGEN). cDNAs were used for multiplex PCR according to the manufacturer's recommendations (QIAGEN) and quantitative 
real-time PCR using a Power SYBR Green mixture on an AB7300 apparatus (APPLIED BIOSYSTEMS). PCR primer sequences and specific PCR conditions are available upon request.

\section{Sodium bisulfite conversion}

Genomic DNA was isolated from cell lines using the QIAmpDNA Mini Kit (QIAGEN). Extracted DNA was measured using a Nanodrop ${ }^{\circledR}$ Spectrophotometer and $1 \mu \mathrm{g}$ DNA was modified by sodium bisulfite using an EZ DNA Methylation ${ }^{\mathrm{TM}}$ Kit (ZYMO RESEARCH) according to the manufacturer's recommendations.

\section{Methylation-Specific PCR (MSP) and quantitative MSP (qMSP) amplification}

After sodium bisulfate conversion, the recovered DNA template was quantified using a Nanodrop ${ }^{\circledR}$ Spectrophotometer and 50ng DNA were used for fluorescence-based, qRT-PCR amplification as introduced above. The PCR primers were specifically designed for bisulfite converted DNA sequence, with sets of primers distinguishing fully methylated, fully unmethylated and wild-type DNA for the PLS3 gene and an internal reference set for the MYOD1 gene control for input DNA as previously described $[14,15]$. The methylated and unmethylated primers were designed with Methyl Primer Express ${ }^{\circledR}$ Software (APPLIED
BIOSYSTEMS) in order to overlap four potential CpG dinucleotide sites. Quantitative SyBR Green PCR reactions were simultaneously performed with primers designed for the bisulfiteconverted methylated, unmethylated and wild-type sequences of PLS3 and with MYOD1 reference primers. The designed pairs of primer sequences were as follows: PLS3 Wild-type sense 5'-AACTTCCCTCTGTCGTCCTTCTC-3', anti-sense 5'-AGGAAATCCCGGAGCCAG-3'; PLS3 Methylated sense 5'-GTTCGGATT TAGGAAATTTC-3', anti-sense 5'-TCGTATCCTTCCCTCGAC-3', PLS3 Unmethylated sense 5'-TTTTGTTTGGATTTAGGAAATTTT-3', anti-sense 5'-CCTCATATCCTTCCCTC A-ACC-3'; MYOD1 sense 5'-CCAACTCCAAATCCCCTCTCTAT3', anti-sense 5'-TGATT AATTTAGATT-GGGTTTAGAGAAGGA-3'. Positive controls were generated by bisulfite conversion of $\mathrm{CpGenome} \mathrm{T}^{\mathrm{TM}}$ Universal Methylated DNA (CHEMICON), a commercially available enzymatically methylated human male genomic DNA used as a methylation-positive control for gene methylation studies. The thermocycling program included: an initial denaturation at $95^{\circ} \mathrm{C}$ for $10 \mathrm{~min}, 40$ cycles of denaturation at $95^{\circ} \mathrm{C}$ for $15 \mathrm{~s}$, annealing at $60^{\circ} \mathrm{C}$ for 60 s and elongation at $72^{\circ} \mathrm{C}$ for $30 \mathrm{~s}$. Each assay was run in duplicate. Reference gene MYOD1 was quantified to normalize mRNA level between cell line samples.
A

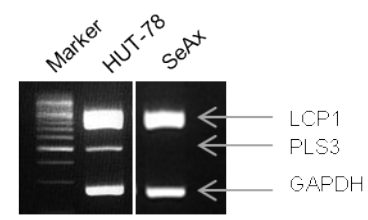

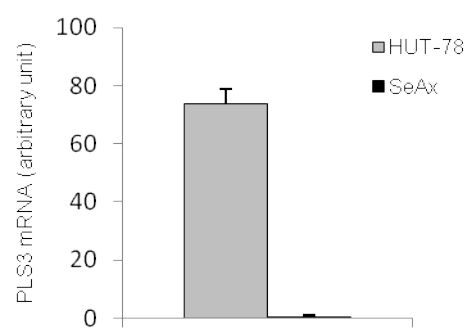

B

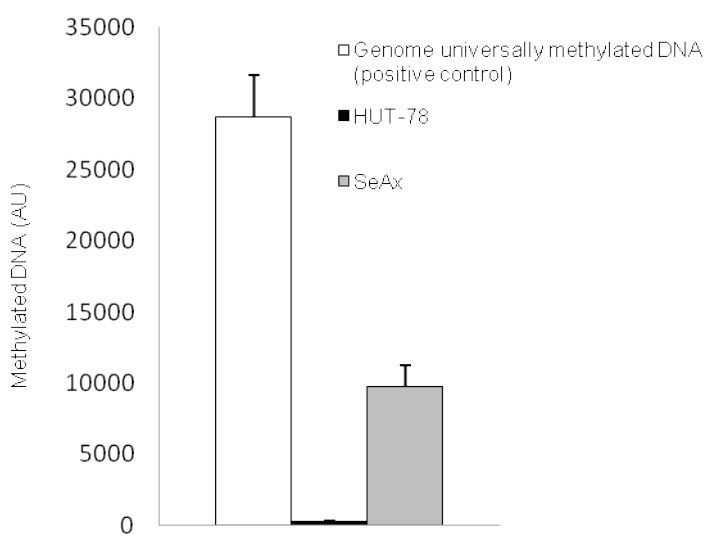

Figure 1: Expression of $P L S 3$ gene and methylation status of a portion of CpG island in HuT-78 and SeAx cell lines.

(A) Expression of PLS3 mRNA levels in HuT-78 and SeAx cells. Basal expression levels of $P L S 3$ and $L C P 1$ transcripts were determined by multiplex PCR and normalized to GAPDH (left panel). PLS3 expression levels were assessed by qRT-PCR (right panel): quantification of the target gene expression was done using the comparative cycle threshold $(C \mathrm{t}$ ) method according to the manufacturer's instructions (Applied Biosystems). An average $C \mathrm{t}$ was calculated for the duplicate reactions and normalized to GAPDH (_Ct $=C \mathrm{t}$ sample $-C \mathrm{t}$ GAPDH). The obtained DCts values are expressed as arbitrary units (A.U.) and presented as mean \pm SD ( $n=3)$.

(B) Methylation status of HuT-78 and SeAx cell lines. qMSP analysis Genomic purifed DNA was treated with sodium bisulfite as briefly described in material and methods. Modified bisulfite DNA was amplified using PLS3-methylated primers and wild-type to ensure complete bisulfite conversion. Positive controls were generated by bisulfite modifying CpG universally methylated DNA. The results were normalized with MYOD1 and presented as mean $\pm \mathrm{SD}(\mathrm{n}=3)$ in arbitrary units (A.U.). 


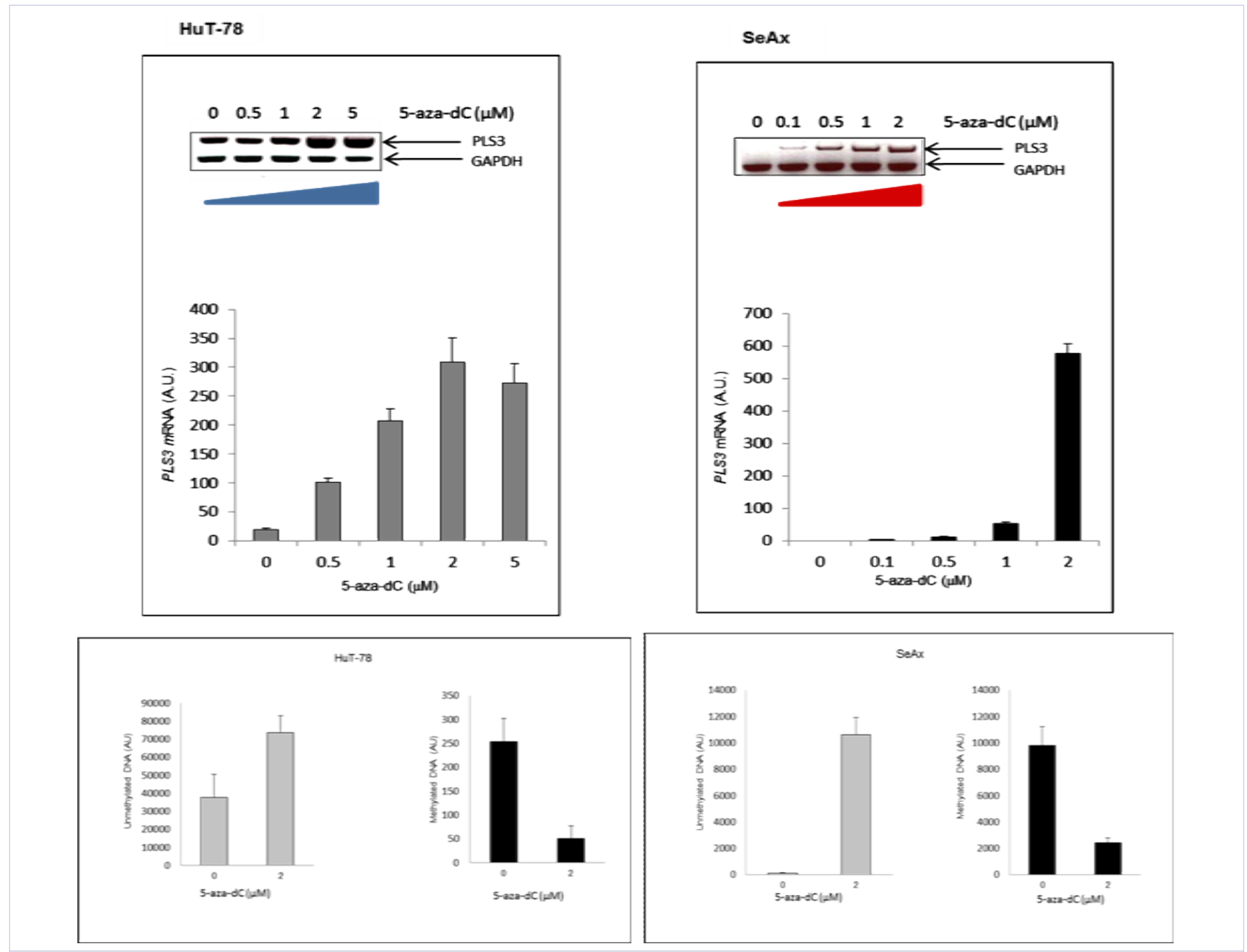

Figure 2: PLS3 mRNA expression (Upper panel) and Methylation status of a portion of CpG island (4 CpG dimers) in the human PLS3 gene (Lower panels) in HuT-78 (A) and SeAx(B) cell lines after treatment with the DNA methyltranferase inhibitor 5-aza-dC.

Upper panels: Cells were exposed or not to increasing concentrations of 5-aza-dC for 6 days. Transcripted PLS3 normalized to GADPH mRNA was determined by multiplex PCR or qRTPCR.

Lower panels: Genomic DNA was isolated from cell lines using the QIAmpDNA Mini Kit (QIAGEN). Extracted DNA was measured using a Nanodrop® Spectrophotometer and $1 \mu \mathrm{g}$ DNA was modified by sodium bisulfite using an EZ DNA Methylation ${ }^{\text {TM }}$ Kit (ZYMO RESEARCH) according to the manufacturer's recommendations.

Modified bisulfite DNA was amplified with PLS3-Unmethylated and PLS3-methylated primers and wild-type to ensure complete bisulfite conversion. The results were normalized with MYOD1.

Results from qMSP analysis are expressed as levels of unmethylated DNA (left) and methylated DNA (right) detected in HuT-78 cells (A) or SeAx cells (B) either unexposed ( 0 ) or exposed to 5 aza- $\mathrm{dC} 2 \mu \mathrm{M}$ for 6 days and presented as mean $\pm \mathrm{SD}(\mathrm{n}=3)$ in arbitrary units (A.U.).

\section{Treatment with 5-aza-2'-deoxycytidine (5-aza-dC)}

HuT-78 and SeAx cells were seeded at the concentration of $5 \times 10^{4}$ cells $/ \mathrm{mL}$ and incubated in a culture medium with or without 5 -aza-dC (SIGMA) at a concentration of $0.1,0.5,1,2$ or $5 \mu \mathrm{M}$ for 6 days with daily medium changes and fresh 5 -aza-dC addition every day. Total RNA or DNA from control and treated cells was analyzed by multiplex PCR and qRT-PCR.

\section{Results and Discussion}

\section{Methylation status}

Our present results obtained by quantitative MSP analysis revealed a quantitative relationship between the methylation status of the PLS3 CpG region and PLS3 expression in HuT-78 and SeAx Sezary cell lines. As shown in figure 1, hypomethylation of PLS3 promoter was detected in HuT-78 cell line and related to the significant expression of PLS3 mRNA, which was detected by multiplex PCR and qRT-PCR. In contrast, hypermethylation of PLS3 promoter was observed in SeAx cell line and this could be associated with undetectable PLS3 mRNA.

\section{Rescue of PLS3 expression by epigenetic 5-Aza-dC exposure}

To further examine whether the silencing of PLS3 expression 
in SeAx cells was attributed to the methylation status of the promoter and whether PLS3 synthesis could be restored by treatment with DNA methylation inhibitors, SeAx cells were exposed to 5-aza-dC for 6 days and then analyzed for their methylation status by qMSP and for PLS3 expression by qRT-PCR. As control, we similarly treated HuT-78 cells that constitutively expressed PLS3. As shown in figure 2, PLS3 promoter hypomethylation and gene expression was induced in SeAx cells after the 5 -aza-dC treatment according to a dosedependent manner, with a maximal expression for $2 \mu \mathrm{M} 5$-aza-dC. Of interest, PLS3 promoter hypomethylation and gene expression in HuT-78 cells was increased by 5 -aza-dC treatment, with a maximal expression with $2 \mu \mathrm{M}$ inhibitor. These data indicate that hypomethylation of the PLS3 promoter is linked to constitutive PLS3 gene expression in HuT-78 cell line and that PLS3 promoter methylation controls gene expression in SeAx cells.

During the last decade, substantial evidence has demonstrated the importance of epigenetic mechanisms in the transcriptional regulation of genes that play critical roles in the process of cancer progression [16]. Some cancers display aberrant methylation profiles in multiple genes and global genomic hypomethylation have been described in several malignant cancers.

In the present study, PLS3 mRNA expression was confirmed to be associated with aberrant promoter hypomethylation in HuT-78 CTCL cell line and was increased by treatment with DNA methylation inhibitors such as 5-aza-dC. Significant PLS3 expression in SeAx cells that do not constitutively express PLS3 was also induced by treatment with 5-aza-dC.

A hallmark of cancer is a paradoxical aberration of DNA methylation patterns, with a global loss of DNA methylation that coexists with regional hypermethylation of certain genes [17]. It has been proposed that hypermethylation and hypomethylation in cancers are independent processes, which target different programs at different stages in tumorigenesis. Hypermethylation and silencing of genes that regulate proliferation were proposed to be critical for deregulation growth early in carcinogenesis, while hypomethylation and activation of other genes may be more important for metastasis [13]. Activation of PLS3 expression in hematopoietic cells through alteration of DNA methylation may play a role in cutaneous T-cell lymphoma oncogenesis and tissue invasion by modulating actin cytoskeleton, as suggested by our recent data demonstrating that PLS3 expression favors tumor cell migration [9].

In conclusion, our results confirmed that an epigenetic mechanism regulates PLS3 expression in CTCL as shown by PLS3 promoter hypomethylation status in CTCL lines. As PLS3 expression has potential as molecular biomarker in Sezary syndrome, it might be suggested that methylation status may serve as a novel biomarker in malignant T-cells from SS patients.

\section{References}

1. Kari L, Loboda A, Nebozhyn M, Rook AH, Vonderheid EC, et al. (2003) Classification and prediction of survival in patients with the leukemic phase of cutaneous T cell lymphoma. J Exp Med 197: 1477-1488.

2. Nebozhyn M, Loboda A, Kari L, Rook AH, Vonderheid EC, et al. (2006) Quantitative PCR on 5 genes reliably identifies CTCL patients with 5\% to $99 \%$ circulating tumor cells with $90 \%$ accuracy. Blood 107: 3189 3196.

3. van Doorn R, Dijkman R, Vermeer MH, Out-Luiting JJ, van der RaaijHelmer EM, et al. (2004) Aberrant expression of the tyrosine kinase receptor EphA4 and the transcription factor twist in Sezary syndrome identified by gene expression analysis. Cancer Res 64: 5578-5586.

4. Su MW, Dorocicz I, Dragowska WH, Ho V, Li G, et al. (2003) Aberrant expression of T-plastin in Sezary cells. Cancer Res 63: 7122-7127.

5. Booken N, Gratchev A, Utikal J, Weiss C, Yu X, et al. (2008) Sezary syndrome is a unique cutaneous T-cell lymphoma as identified by an expanded gene signature including diagnostic marker molecules CD01 and DNM3. Leukemia 22: 393-399.

6. Capriotti E, Vonderheid EC, Thoburn CJ, Wasik MA, Bahler DW, et al. (2008) Expression of T-plastin, FoxP3 and other tumor-associated markers by leukemic T-cells of cutaneous T-cell lymphoma. Leuk Lymphoma 49: 1190-1201.

7. Tang N, Gibson H, Germeroth T, Porcu P, Lim HW, et al. (2010) T-plastin (PLS3) gene expression differentiates Sezary syndrome from mycosis fungoides and inflammatory skin diseases and can serve as a biomarker to monitor disease progression. Br J Dermatol 162: 463-466.

8. Jones CL, Ferreira S, McKenzie RC, Tosi I, Caesar JA, et al. (2012) Regulation of T-plastin expression by promoter hypomethylation in primary cutaneous T-cell lymphoma. J Invest Dermatol 132: 20422049.

9. Begue E, Jean-Louis F, Bagot M, Jauliac S, Cayuela JM, et al. (2012) Inducible expression and pathophysiologic functions of T-plastin in cutaneous T-cell lymphoma. Blood 120: 143-154.

10. Oprea GE, Krober S, McWhorter ML, Rossoll W, Muller S, et al. (2010) Plastin 3 is a protective modifier of autosomal recessive spinal muscular atrophy. Science 320: 524-527.

11. Lin CS, Park T, Chen ZP, Leavitt J(1993) Human plastin genes. Comparative gene structure, chromosome location, and differential expression in normal and neoplastic cells. J Biol Chem 268: 27812792.

12. Lin CS, Lau A, Huynh T, Lue TF (1999) Differential regulation of human T-plastin gene in leukocytes and non-leukocytes: identification of the promoter, enhancer, and CpG island. DNA Cell Biol 18: 27-37.

13. Szyf M, Pakneshan P, Rabbani SA (2004) DNA demethylation and cancer: therapeutic implications. Cancer Lett 211: 133-143.

14. Eads CA, Danenberg KD, Kawakami K, Saltz LB, Blake C, et al. (2000) MethyLight: a high-throughput assay to measure DNA methylation. Nucleic Acids Res 28: E32.

15. Trinh BN, Long TI, Laird PW (2001) DNA methylation analysis by MethyLight technology. Methods 25: 456-462.

16. Rodriguez-Paredes M, Esteller M (2011) Cancer epigenetics reaches mainstream oncology. Nat Med 17: 330-339.

17. Ehrlich M (2002) DNA methylation in cancer: too much, but also too little. Oncogene 21: 5400-5413. 